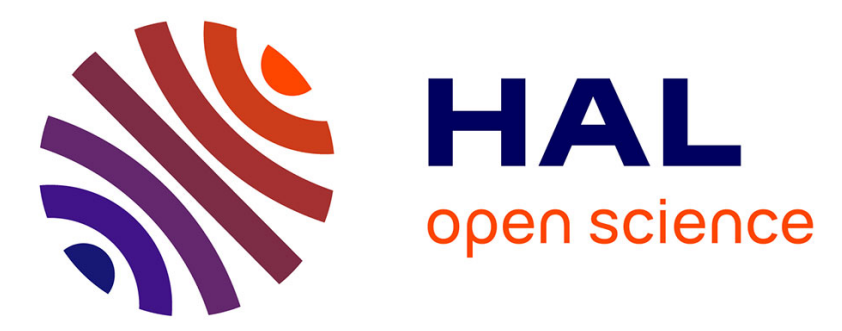

\title{
A New Seven Degrees-of-Freedom Parallel Robot With a Foldable Platform
}

\author{
Wissem Haouas, Redwan Dahmouche, Nadine Piat, Guillaume Laurent
}

\section{To cite this version:}

Wissem Haouas, Redwan Dahmouche, Nadine Piat, Guillaume Laurent. A New Seven Degrees-ofFreedom Parallel Robot With a Foldable Platform. Journal of Mechanisms and Robotics, 2018, 10 (4), pp.1 - 15. 10.1115/1.4039693 . hal-02399249

\section{HAL Id: hal-02399249 https://hal.science/hal-02399249}

Submitted on 9 Dec 2019

HAL is a multi-disciplinary open access archive for the deposit and dissemination of scientific research documents, whether they are published or not. The documents may come from teaching and research institutions in France or abroad, or from public or private research centers.
L'archive ouverte pluridisciplinaire HAL, est destinée au dépôt et à la diffusion de documents scientifiques de niveau recherche, publiés ou non, émanant des établissements d'enseignement et de recherche français ou étrangers, des laboratoires publics ou privés. 


\title{
A New Seven Degrees-of-Freedom Parallel Robot With a Foldable Platform
}

\author{
Wissem Haouas \\ FEMTO-ST Institute \\ Univ. Bourgogne Franche-Comté \\ Besançon, France \\ Email: wissem.haouas@femto-st.fr
}

\author{
Redwan Dahmouche * \\ FEMTO-ST Institute \\ Univ. Bourgogne Franche-Comté \\ Besançon, France \\ Email: redwan.dahmouche@femto-st.fr
}

\author{
Nadine Le Fort-Piat \\ FEMTO-ST Institute \\ Univ. Bourgogne Franche-Comté \\ Besançon, France \\ Email: nadine.piat@ens2m.fr \\ Guillaume J. Laurent \\ FEMTO-ST Institute \\ Univ. Bourgogne Franche-Comté \\ Besançon, France \\ Email: guillaume.laurent@ens2m.fr
}

\begin{abstract}
This paper presents a new parallel robot with an integrated gripper. The grasping capability of the robot is obtained by a foldable platform that can be fully controlled by actuators located on the base of the seven degrees of freedom (DoF) parallel structure. This mechanism combines three key specificities in robotics which are: compactness, rigidity, and high blocking forces. The paper presents the new structure, its kinematic modeling and an analysis of its workspace and grasping force capabilities. In addition, a prototype is presented and tested in manipulation and insertion operations, which validates the proposed concept.
\end{abstract}

Keywords: Parallel robots, Configurable Platform, Grasping, Redundantly Actuated Robot.

\section{Introduction}

Parallel robots are closed-loop mechanisms composed of a moving platform connected to the base by independently actuated serial kinematic chains [1]. This class of robots has drawn the attention of the industrial and academic communities due to their notable characteristics. Indeed, compared to serial robots, parallel mechanisms (PMs) have the advantages of high stiffness, load-to-weight ratio, and dynamics. Indeed, industrial robots such as the Gough-Stewart [2] or Delta [3] have been used in flight simulation and high-speed pick-and-place systems respectively, where $20 \mathrm{G}$ accelerations can be exceeded [4] [5] [6].

The high stiffness property also allows to design highly compact structures with all actuators placed on the robot base [7] [8] [9] [10]. However, PMs suffer from more kinematic singularities, limited workspaces and complex kinematic models [1]. One solution to moderate these drawbacks is to use actuation and sensing redundancy which can be exploited to reduce singularities [11] [12] [13] and to improve the kinematic manipulability and dexterity as shown in [14] [15].

In order to manipulate objects, the solution generally used to provide grasping capabilities is to attach a gripper on the robot's platform [16] [17]. However, in addition to the tool weight added to the platform which

*Address all correspondence to this author. 
typically slows down the robot speed, the extra gripper's volume and its wiring (power and/or signal) might be prohibitive in some application where high compactness and high grasping/cutting forces are required [18].

An original approach to eliminate these drawbacks is to integrate the grasping functionality in the same structure as the robot platform. This original concept relies on a parallel structure with a configurable platform. Parallel robots with configurable platforms are PMs whose platforms have at least one additional mobility, usually obtained by an inner closed loop chain [19]. A relatively few articles have been published on topics related to this class of robots. Some of these architectures use configurable platforms to provide rotation to the end-effector, such as the Par4 robot [20], which is a commercially available robot on the market known as the Adept Quattro. The configurable platform can also be used for grasping such as in [21], where a platform consisting of a planar parallelogram mechanism was used as a gripper. In 2005, Mohamed and Gosselin analyzed the kinematics of several designs of both planar and spatial parallel robots with configurable platforms by means of screw theory [22]. Lambert developed and fabricated, respectively, in 2014 and 2015, a five-DoF parallel manipulator that generates three translations, one rotation plus a linear grasping motion [23] and a redundant parallel haptic interface whose platform has six DoFs for full motion control and one DoF for grasping [24].

Thus, instead of appending an additional gripper to perform grasping tasks, which reduces the robot dynamics, and compactness, a configurable robot platform that acts as a gripper can be preferred. In addition, no wiring or tubing to transmit power and signals is required to actuate the gripper, which makes the robot even more compact and facilitates its integration.

To fulfill the objective of designing a compact and stiff robot with grasping capabilities, this paper presents a new seven DoFs redundant parallel mechanism with a configurable platform. This structure is actuated with eight linear positioners located on the robot base that control the 6-DoFs robot platform position and orientation as well as the grasping functionality. The actuation redundancy could be used to reduce singularities, enlarge the robot workspace, enhance the robot dynamics and increase the grasping force.

The major novelty in the proposed structure compared to existing parallel robots with configurable platforms is the usage a simple foldable platform instead of more complex closed-loop chains, as presented in the aforementioned papers. To the best of our knowledge, this is the first 7-DoF parallel robot with a foldable platform. This additional DoF can be used to provide grasping or cutting capabilities via two fingers or scissors located on both sides of the foldable platform. This structure is an extension of a previous concept designed for minimally invasive surgery with four DoFs (three rotations with gripping function) [25].

The next section of the paper presents the kinematic structure of the robot. Then, its kinematic modeling is presented in Section 3 and analyzed regarding the workspace and the grasping forces in Section 4. The experimental proof-of-concept of the robot is demonstrated and its grasping and manipulation capabilities validated in section 5. Finally, the two last sections present a discussion about the robot capabilities and limits and some perspectives to improve its design and control.

\section{$2 \quad$ Kinematic Structure}

The concept of the proposed structure is described in the architectural scheme illustrated in Fig. 1, where joints are represented by rectangles, and links between those joints are represented by lines.

The robot is actuated using eight moving stages, symmetrically fixed on the base. Each moving stage supports one strut, which is attached to the platform and to the moving stage by mean of two spherical joints. The right part of the top platform is attached to the left one through a revolute joint in order to generate a folding mobility.

This design allows six-DoF motion and the folding of the top platform. The configuration of the platform is defined by the coordinates $(x, y, z)$ of the point $O_{p}$ located in the center of the platform while three Euler angles $(\alpha, \beta, \gamma)$ are used to describe the relative orientation of the platform with the base, as represented in Fig. 2. The relative angle between the two parts of the foldable platform is represented by $\theta$.

The device is redundantly actuated since the seven DoFs of the architecture are controlled by eight independent actuators.

\section{Kinematic Modeling}

\subsection{Geometry of the Manipulator}

To describe the geometry of the kinematic model, we define the ensuing parameters. The global coordinate frame $\mathcal{F}_{w}=\left(O_{w}, \mathbf{x}_{w}, \mathbf{y}_{w}, \mathbf{z}_{w}\right)$ is set at the robot base. Every strut has a total length $l_{i}$, where $i=1, \ldots, 8$ denotes the leg number. Each of them is attached on one end to the top platform through a spherical joint $A_{i}$ and attached to the base plates on the other end via a spherical joint $B_{i}$. The configurable platform carries the two fingers of the gripper. The moving plate is attached to a positioning stage $q_{i}$ fixed vertically on the base. Each of these 




Figure 1. Architectural scheme of the manipulator. The blocks $\mathrm{P}, \mathrm{R}$, and $\mathrm{S}$ represent prismatic, revolute, and spherical joints, respectively.

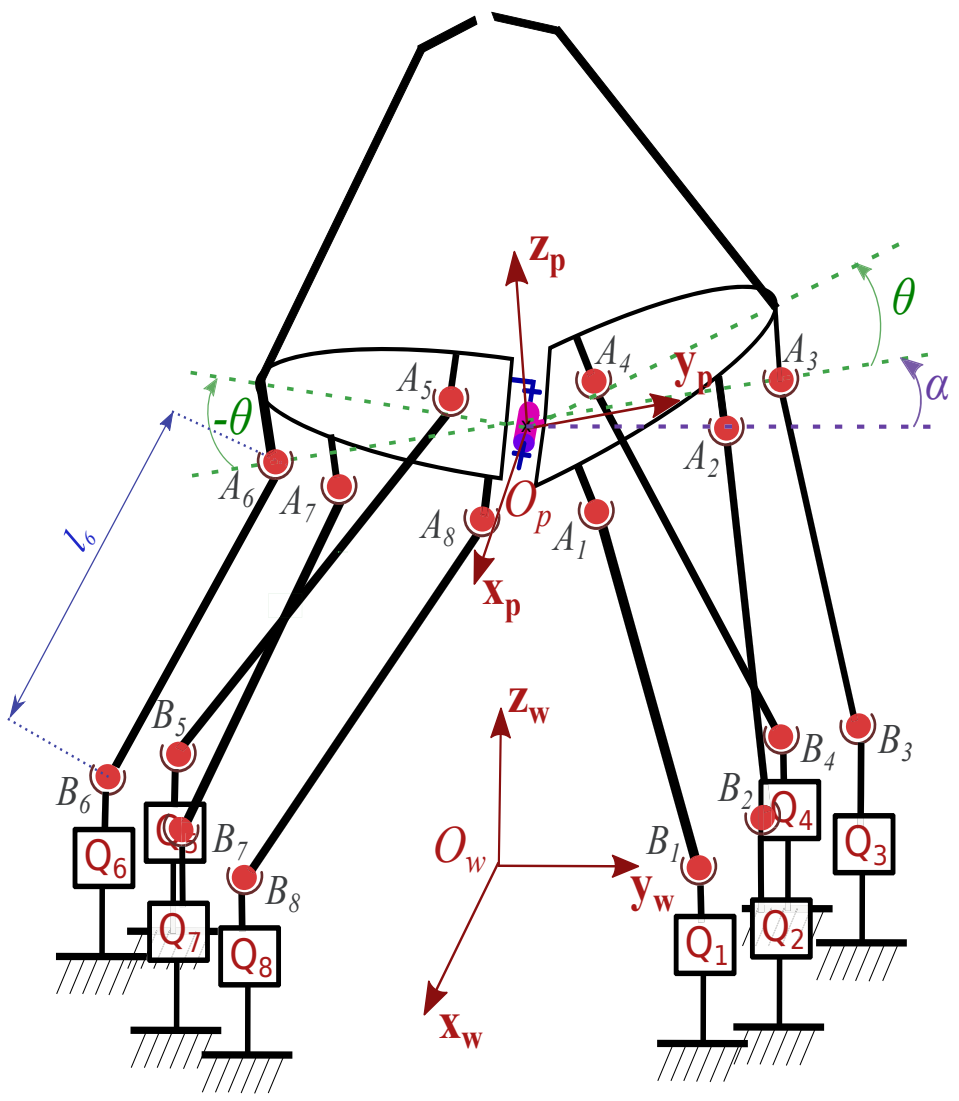

Figure 2. The kinematic architecture of the robot.

stages can move $B_{i}$ vertically (see Fig. 2).

The left part of the configurable platform is attached to the right one through a revolute joint along $\mathbf{x}_{p}$ located in $O_{p}$, where $\theta_{p}$ denotes the relative rotation angle between the two parts. $\mathcal{F}_{r}=\left(O_{p}, \mathbf{x}_{p}, \mathbf{y}_{r}, \mathbf{z}_{r}\right)$ and $\mathcal{F}_{l}=\left(O_{p}, \mathbf{x}_{p}, \mathbf{y}_{l}, \mathbf{z}_{l}\right)$ are the frames attached to the right and the left parts of the platform, respectively. Finally, $\mathcal{F}_{p}=\left(O_{p}, \mathbf{x}_{p}, \mathbf{y}_{p}, \mathbf{z}_{p}\right)$ represents the frame defined by $O_{p}, \mathbf{x}_{p}$ and the symmetrical axis $\mathbf{z}_{p}$ between the left and the right parts of the platform.

\subsection{Inverse Kinematics}

The inverse kinematics aims to find the position of the eight linear actuators, ${ }^{w} B_{i}$ for $i=1, \ldots, 8$, for a given pose of the top platform. The input variables are the folding angle $\theta_{p}$, the position of the platform ${ }^{w} O_{p}$ and the 
rotation matrix ${ }^{w} \mathbf{R}_{p}$ between the moving frame $\mathcal{F}_{p}$ and the reference frame $\mathcal{F}_{w}$.

We define respectively ${ }^{p} \mathbf{R}_{r}$ and ${ }^{p} \mathbf{R}_{l}$ as the rotation matrices of the moving frames $\mathcal{F}_{r}$ and $\mathcal{F}_{l}$ with respect to the frame $\mathcal{F}_{p}$, where:

$$
\left\{\begin{array}{l}
{ }^{p} \mathbf{R}_{r}=\operatorname{rot}\left(\mathbf{x}_{p}, \theta_{p}\right) \\
{ }^{p} \mathbf{R}_{l}=\operatorname{rot}\left(\mathbf{x}_{p},-\theta_{p}\right)
\end{array}\right.
$$

We denote respectively ${ }^{w} A_{i},{ }^{r} A_{i}$ and ${ }^{l} A_{i}$ the coordinates of the point $A_{i}$ expressed in $\mathcal{F}_{w}, \mathcal{F}_{r}$ and $\mathcal{F}_{l}$. The positions of the eight spherical joints attached to the top platform are given by:

$$
\left\{\begin{array}{l}
{ }^{w} A_{i}={ }^{w} \mathbf{R}_{r}{ }^{r} A_{i}+{ }^{w} O_{p}={ }^{w} \mathbf{R}_{p}{ }^{p} \mathbf{R}_{r}{ }^{r} A_{i}+{ }^{w} O_{p}, \quad i=1, \ldots, 4 \\
{ }^{w} A_{i}={ }^{w} \mathbf{R}_{l}{ }^{l} A_{i}+{ }^{w} O_{p}={ }^{w} \mathbf{R}_{p}{ }^{p} \mathbf{R}_{l}{ }^{l} A_{i}+{ }^{w} O_{p}, \quad i=5, \ldots, 8
\end{array}\right.
$$

The loop-closure constraint equations are obtained from the distance constraint between the points ${ }^{w} A_{i}$ and ${ }^{w} B_{i}$, representing the constant length of the struts (i.e the vector $A_{i} B_{i}$ norm):

$$
\left\|^{w} B_{i}-{ }^{w} A_{i}\right\|=l_{i}, \quad i=1, \ldots, 8
$$

In the case of vertical stages, Equation (3) can be rewritten using the points Cartesian coordinates as follows:

$$
\left(b_{x i}-a_{x i}\right)^{2}+\left(b_{y i}-a_{y i}\right)^{2}+\left(b_{z i}+q_{i}-a_{z i}\right)^{2}=l_{i}^{2}, \quad i=1, \ldots, 8
$$

Equation (4) gives two solutions of the actuators positions $q_{i}$.

\subsection{Inverse Jacobian Matrix}

The instantaneous motion of the platform is obtained by taking the derivative of the kinematic Equation (3) with respect to time,

$$
\left\{\begin{array}{r}
\left({ }^{w} B_{i}-{ }^{w} A_{i}\right)^{t}\left([\Omega]_{\times}{ }^{w} \mathbf{R}_{r}{ }^{r} A_{i}+{ }^{w} \mathbf{R}_{p}\left[{ }^{p} \mathbf{x}_{p}\right] \times \omega_{\theta}{ }^{p} \mathbf{R}_{r}{ }^{r} A_{i}+{ }^{w} \mathbf{v}_{p}\right) \\
=\left({ }^{w} B_{i}-{ }^{w} A_{i}\right){ }^{t} \dot{\mathbf{b}}_{i}, \quad i=1, \ldots, 4 \\
\left({ }^{w} B_{i}-{ }^{w} A_{i}\right){ }^{t}\left([\Omega]_{\times}{ }^{w} \mathbf{R}_{l}{ }^{l} A_{i}-{ }^{w} \mathbf{R}_{p}\left[{ }^{p} \mathbf{x}_{p}\right] \times \omega_{\theta}{ }^{p} \mathbf{R}_{l}{ }^{l} A_{i}+{ }^{w} \mathbf{v}_{p}\right) \\
=\left({ }^{w} B_{i}-{ }^{w} A_{i}\right){ }^{t} \dot{\mathbf{b}}_{i}, \quad i=5, \ldots, 8
\end{array}\right.
$$

Here, $\dot{\mathbf{b}}_{i}=\left[\begin{array}{lll}0 & 0 & \dot{q}_{i}\end{array}\right]^{t}$ is the instantaneous velocity of points ${ }^{w} B_{i}$, where $\dot{q}_{i}$ represents the vertical speed of an actuator. In Equation (5), $\Omega=\left[\omega_{\alpha} \omega_{\beta} \omega_{\gamma}\right]^{t}$ is the instantaneous angular velocity vector of the foldable platform when $\theta=0$, and $\omega_{\theta}$ is the instantaneous angular velocity between the two parts of the foldable platform. The symbol $[\Omega]_{\times}$ represents the cross product skew matrix, and ${ }^{w} \mathbf{v}_{p}=\left[v_{x} v_{y} v_{z}\right]^{t}$ represents the velocity of the point $O_{p}$ located in the middle of the foldable platform.

We can write Equation (5) in matrix form as:

$$
\mathbf{J}_{l} \mathbf{t}=\mathbf{J}_{r} \dot{\mathbf{q}}
$$

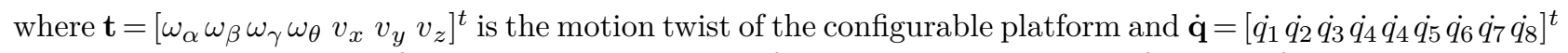
is the actuating displacement of the linear motors. The left matrix $\mathbf{J}_{l,(8 \times 7)}$ has the following form: 


$$
\left[\begin{array}{ccc}
\left(B_{1} A_{1} \times O_{w} A_{1}\right)^{t} & \left(\left(B_{1} A_{1} \times O_{w} A_{1}\right) \cdot \mathbf{x}_{p}\right)^{t} & B_{1} A_{1}{ }^{t} \\
\vdots & & \\
\left(B_{4} A_{4} \times O_{w} A_{4}\right)^{t} & \left(\left(B_{4} A_{4} \times O_{w} A_{4}\right) \cdot \mathbf{x}_{p}\right)^{t} & B_{4} A_{4}{ }^{t} \\
\left(B_{5} A_{5} \times O_{w} A_{5}\right)^{t} & -\left(\left(B_{5} A_{5} \times O_{w} A_{5}\right) \cdot \mathbf{x}_{p}\right)^{t} & B_{5} A_{5}{ }^{t} \\
\vdots & \\
\left(B_{8} A_{8} \times O_{w} A_{8}\right)^{t} & -\left(\left(B_{8} A_{8} \times O_{w} A_{8}\right) \cdot \mathbf{x}_{p}\right)^{t} & B_{8} A_{8}{ }^{t}
\end{array}\right]
$$

where the $\cdot$ and $\times$ operators represent respectively the scalar and cross products. The right matrix $\mathbf{J}_{r,(8 \times 8)}$ has the following form:

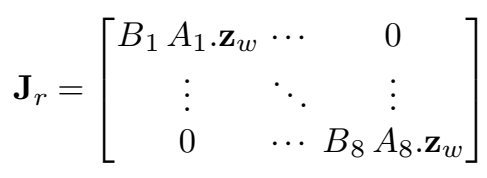

The inverse of the Jacobian matrix relating the eight actuator velocities $\dot{\mathbf{q}}$ to the motion twist $\mathbf{t}$ is:

$$
\dot{\mathbf{q}}=\mathbf{J}_{(8 \times 7)}^{-1} \mathbf{t}=\mathbf{J}_{r}^{-1} \mathbf{J}_{l} \mathbf{t}
$$

The vector $\dot{\mathbf{q}}$ is the image of $\mathbf{t}$ under the transformation $\mathbf{J}^{-1}$. Thus, the mobility of the mechanism is defined by the rank of $\mathbf{J}_{r}$ and $\mathbf{J}_{l}$. Referring to Equation (8), the diagonal matrix $\mathbf{J}_{r}$ becomes rank-deficient only when one of the legs is parallel to the base. Unlike $\mathbf{J}_{r}$, the analytical analysis of $\mathbf{J}_{l}$ is more complex. Nevertheless, the results presented in the next section on the grasping force calculation demonstrates that the rank of the Jacobian matrix is seven $\left(\mathbf{J}^{-T} \mathbf{J}^{-1}\right.$ being invertible) inside the geometrically analyzed workspace. Although, exhaustive singularity analysis requires further investigations.

\section{Design and Analysis of the Mechanism}

\subsection{Mechanical Design of the Robot}

For the sake of simplicity, the kinematic design is explicitly described by a reduced number of parameters under symmetrical assumptions of the robot architecture. Assuming identical lengths of the eight moving struts $l_{i}$, the joints ${ }^{w} A_{i}$ and ${ }^{w} B_{i}$ are respectively symmetrically fixed to the foldable platform and to the mobile actuated bases. By this way, the robot can be fully described using only six parameters $\left(\mathrm{h}_{1}, \mathrm{~h}_{2}, \mathrm{~h}_{3}, \mathrm{~h}_{4}, \phi_{1}\right.$, and $\left.\phi_{2}\right)$ which are shown in Fig. 3 and Fig. 4.

The actuators are placed on the base where space constraints are less critical. Large displacements of the end-effector can then be obtained thanks to high actuator strokes.

Inspired by the works [26] [27] on design of 6-DoF parallel robots, the kinematic parameters were selected to obtain a large workspace and homogeneous performances in its different DoF. First, the impact of the angles $\phi_{1}$ and $\phi_{2}$ on the robot manipulability index and its workspace was studied.

As a reminder, the manipulability is a performance index that indicates the capability of the structure to transmit the actuators motion in all degrees of freedom of the Cartesian space. Furthermore, a high manipulability signals that the robot is far from singular configurations. The manipulability value is calculated through the singular value decomposition (SVD) of the full kinematic Jacobian $\mathbf{J}[28]$.

Figures 5 and 6 show respectively the manipulability index of the robot at its home position and the volume of its workspace as a function of $\phi_{1}$ and $\phi_{2}$. It is noteworthy that these two performance indices are apparently inversely related, which means that a trade-off has to be found.

Moreover, the dimensions of the prototype must take into account the fabrication and assembly constraints. For example, $\phi_{1}$ and $\phi_{2}$ cannot be equal to $0^{\circ}$ or $45^{\circ}$ for the fact that two consecutive joints $A_{i}$ and $A_{i+1}$ or $B_{i}$ and $B_{i+1}$ will be coincident, and this is not feasible in practice. 


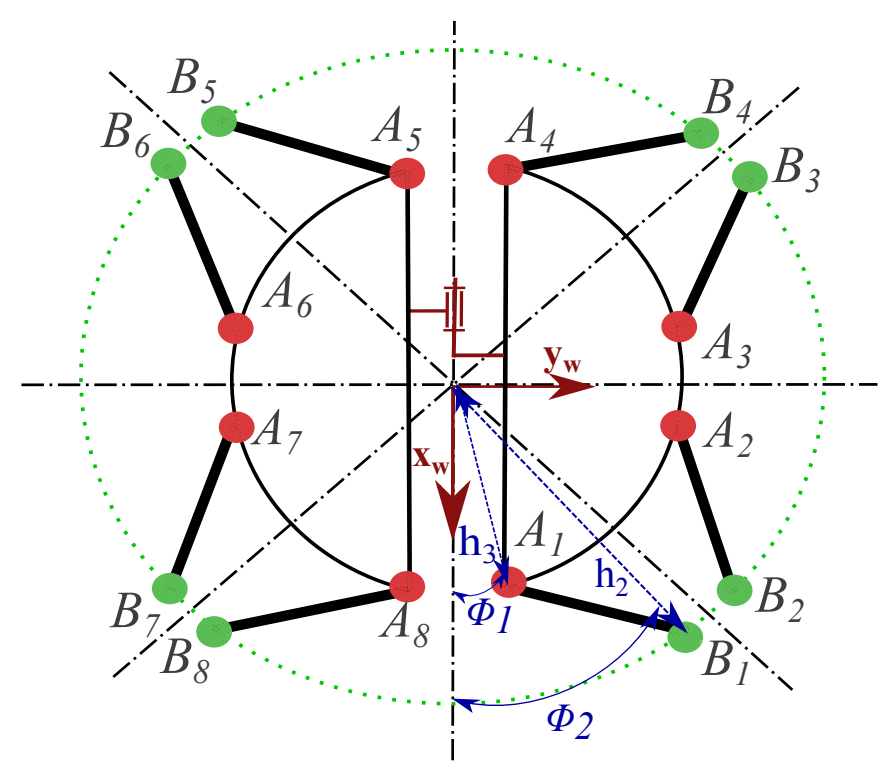

Figure 3. Geometrical description of the top platform and bottom stages.

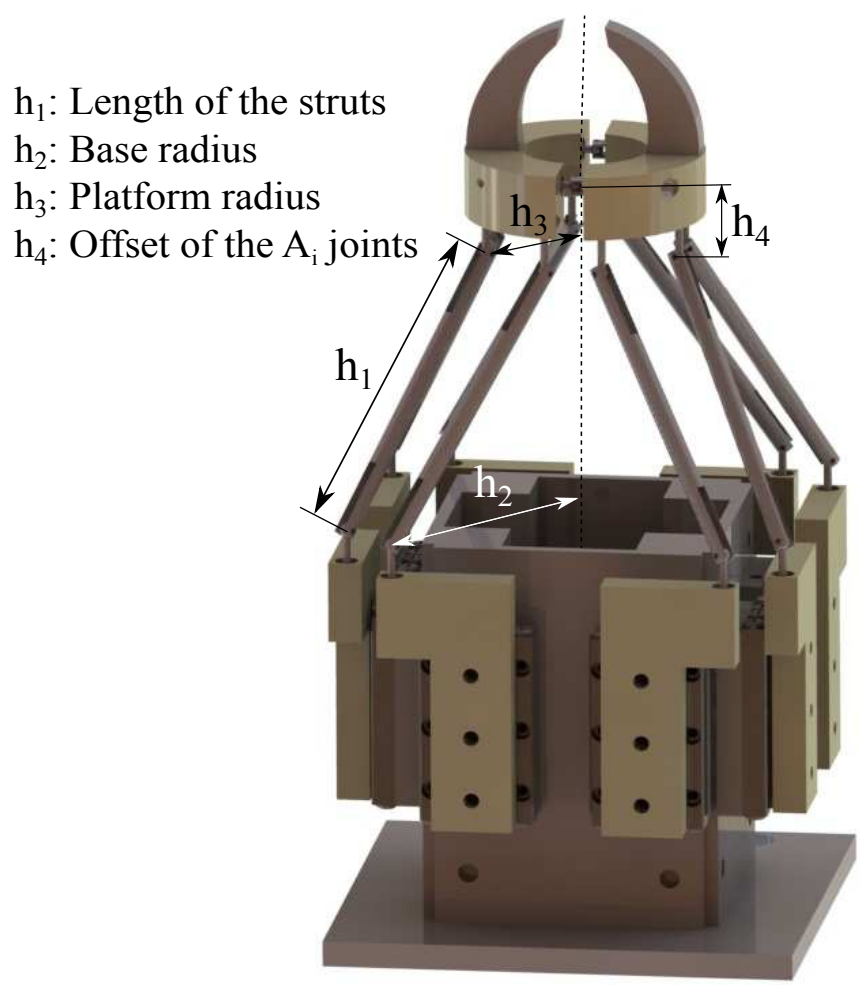

Figure 4. Kinematic design parameters h1, h2, h3 and h4.

Therefore, the values of the chosen parameters $\phi_{1}$ and $\phi_{2}$ are $18^{\circ}$ and $38^{\circ}$, respectively. Accordingly, the corresponding performances are, 651 for the manipulability index and $157 \mathrm{~cm}^{3}$ for the workspace volume.

The ratio of the parameters $\left(\mathrm{h}_{4} / \mathrm{h}_{2}\right)$ affects the reachable workspace in two manners. When this ratio is close to one, the displacements of the manipulator in the $\mathbf{x}$ and $\mathbf{y}$ directions are increased but its rotational mobilities are reduced. The inverse happens when this ratio is close to zero. In both cases, the global manipulability is low. The kinematic design parameters of the prototype, shown in Table 1, have been selected to be close to the mid-ground with respect to the manufacturing constraints. 


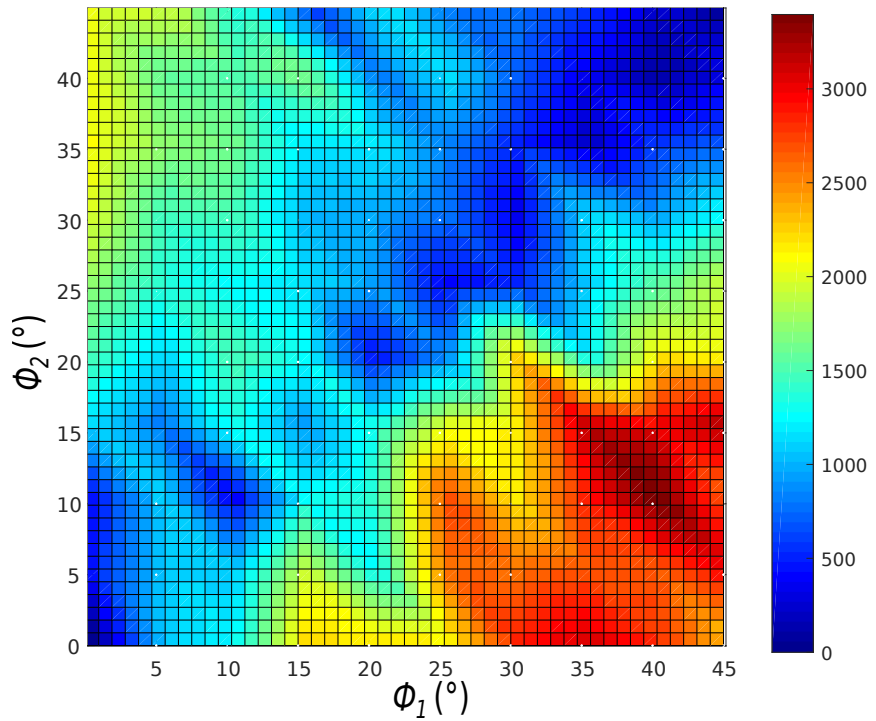

Figure 5. Manipulability index of the manipulator at its home position as a function of $\phi_{1}$ and $\phi_{2}$



Figure 6. Representation of the volume of the workspace $\left(\mathrm{cm}^{3}\right)$ of the manipulator as a function of $\phi_{1}$ and $\phi_{2}$

\subsection{Workspace Analysis}

The workspace of a manipulator can be defined as the volume that the robot can reach. This volume corresponds to the reachable 3D points by the robot end-effector with any orientation of its platform. The mechanical limits of the spherical joints have not been taken into account since they can significantly vary from one design to another.

The theoretical workspace of the presented robot is obtained numerically by considering a travel motion of $18 \mathrm{~mm}$, which corresponds to the travel range of the chosen linear actuators. Obviously, the workspace volume depends also on the length of the robot's fingers. The total volume was evaluated using the six kinematic design parameters presented in Table 1 and the design parameters of the fingers presented in Fig.8. The three-dimensional workspace of the two distal fingers of the manipulator is shown in Fig. 7. The red and the blue ranges show the two reachable spaces of the two fingers of the gripper. With the chosen parameters, the robot's workspace volume is about $157 \mathrm{~cm}^{3}$.

Table 2 shows the maximum displacements, rotation angles, and opening/closing angle of the configurable platform presented in the point $O_{p}$. 
Table 1. Kinematic Design Parameters.

\begin{tabular}{|c|c|c|}
\hline & Parameter & Value \\
\hline $\mathrm{h}_{1}$ & Length of the struts & $50 \mathrm{~mm}$ \\
$\mathrm{~h}_{2}$ & Base radius & $41.5 \mathrm{~mm}$ \\
$\mathrm{~h}_{3}$ & Platform radius & $14.5 \mathrm{~mm}$ \\
$\mathrm{~h}_{4}$ & Offset of the $A_{i}$ joints & $13 \mathrm{~mm}$ \\
$\phi_{1}$ & Angle between $\mathbf{x}_{w}$ and $O_{w} A_{1}$ & $18^{\circ}$ \\
$\phi_{2}$ & Angle between $\mathbf{x}_{w}$ and $O_{w} B_{1}$ & $38^{\circ}$ \\
\hline
\end{tabular}

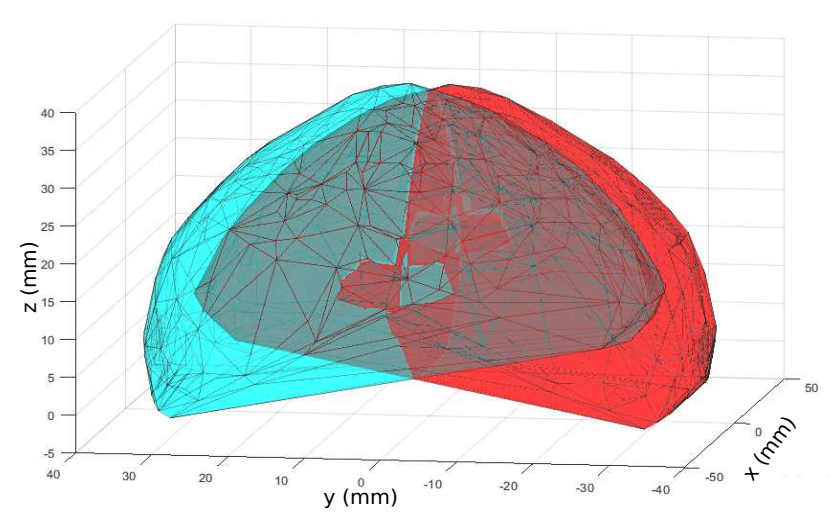

(a) Spacial presentation of the workspace.

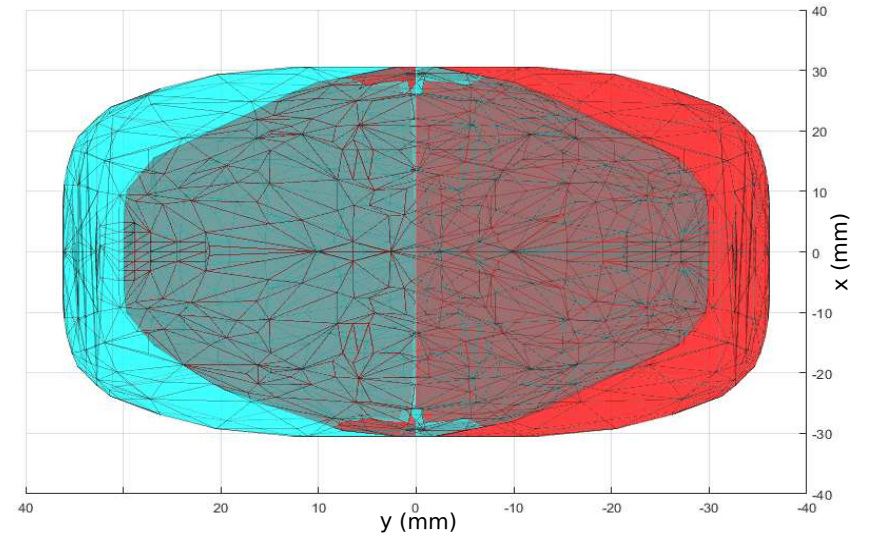

(b) Top view of the workspace.

Figure 7. Theoretical workspace of the two fingers of the manipulator using $18 \mathrm{~mm}$ travel range actuators.

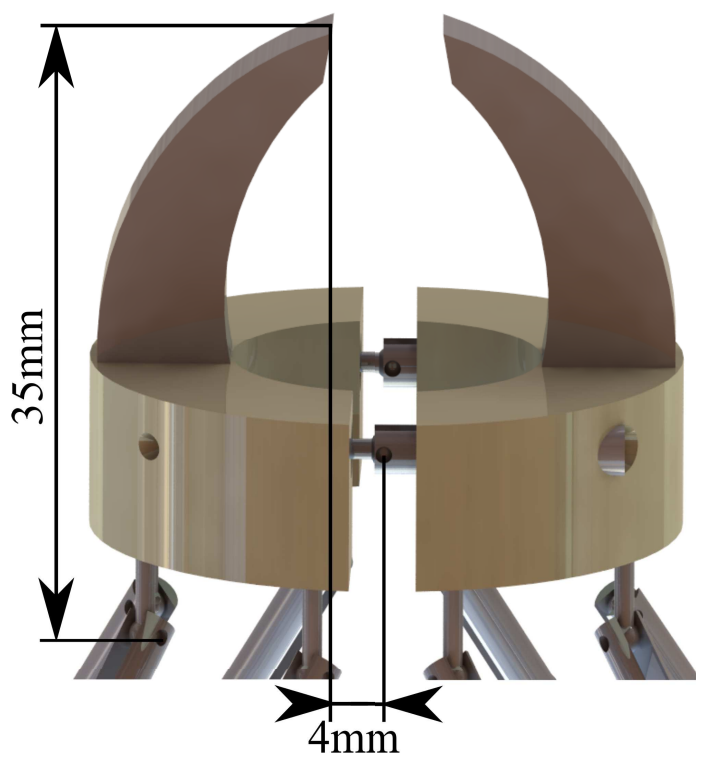

Figure 8. Design parameters of the fingers.

\subsection{Grasping Torque Analysis}

The maximal grasping torque $\tau_{\theta}$ of the end-effector over its workspace can be calculated using the Jacobian matrix. Assuming that the structure has perfect spherical joints, rigid links and that each actuator can generate a force $f$ of $1 \mathrm{~N}$, the static mapping between the actuators force vector $\mathbf{f}$ and the platform's torque $\boldsymbol{\tau}$ is obtained 
Table 2. Maximum displacement and rotation angles of the center of the foldable platform using $18 \mathrm{~mm}$ travel motion of the actuators.

\begin{tabular}{|c|c|c|}
\hline & $\max$ & $\min$ \\
\hline$\alpha(\mathrm{deg})$ & 37 & -37 \\
$\beta(\mathrm{deg})$ & 37 & -37 \\
$\gamma(\mathrm{deg})$ & 42 & -42 \\
$\theta(\mathrm{deg})$ & 37 & -47 \\
$x(\mathrm{~mm})$ & 8 & -8 \\
$y(\mathrm{~mm})$ & 8 & -8 \\
$z(\mathrm{~mm})$ & 9 & -9 \\
\hline
\end{tabular}

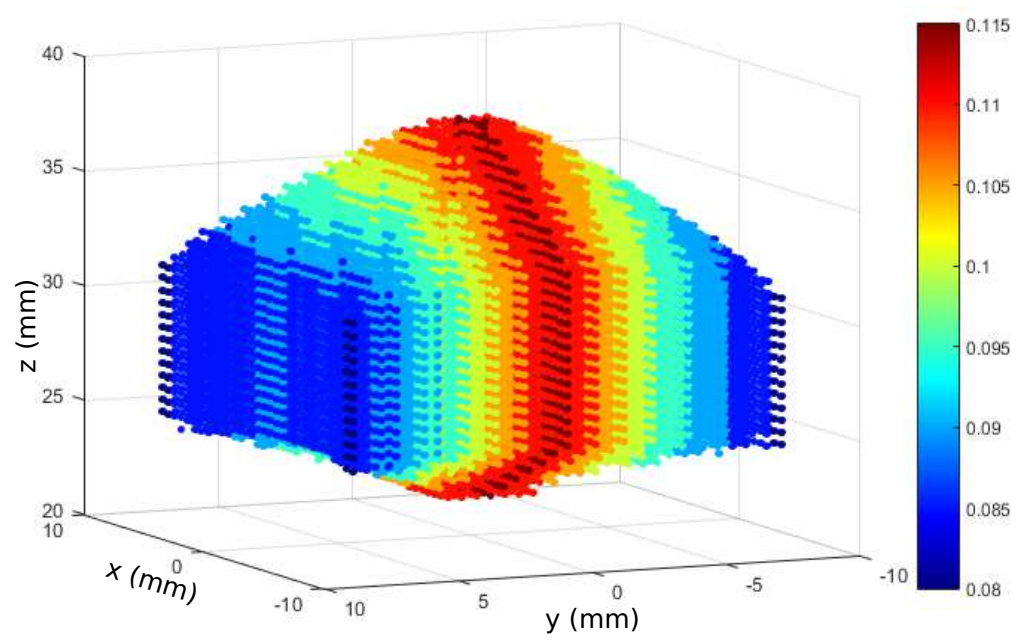

Figure 9. Maximal grasping couple over poses range (N.m).

by [1] ,

$$
\boldsymbol{\tau}=\mathbf{J}^{T} \mathbf{f}
$$

The theoretical grasping torque was evaluated in the reachable workspace of the center of the platform (point $O_{p}$ ) while maintaining the home orientation and folding angle of the platform (i.e, zero rotation and $\theta=0$ ). The direct Jacobian, necessary to estimate the grasping torque, was evaluated from the inverse Jacobian matrix using the Moore-Penrose pseudo-inverse.

The obtained torque over the workspace is shown in Fig. 9. Based on the simulation results, there is no configuration where the manipulator loses the control of the grasping. In fact, the proposed manipulator is able to insure a minimal grasping couple $(80 \mathrm{mN} . \mathrm{m})$ over its workspace which corresponds to a force of $2.8 \mathrm{~N}$ at its fingertips. The maximum $(115 \mathrm{mN} . \mathrm{m})$ and the minimum $(80 \mathrm{mN} . \mathrm{m})$ values obtained are in the same order of magnitude which represent a variation of $\pm 18 \%$ compared to the median value $(97.5 \mathrm{mN} . \mathrm{m}$ ).

It is interesting to note that there is a clear preferable area within the center range of the $\mathbf{y}$ axis where the couple is maximal. Contrarily to the $\mathrm{x}$ direction, the variation of grasping couple along the $\mathbf{y}$ axis is due to the non-symmetrical transmission of the forces applied by the four legs to each part of the platform. Moreover, it can be noted that the grasping torque is constant along the z-axis. This is due to the direction of the legs which remain unchanged when the robot moves vertically. Since the direction of the forces applied on the configurable platform as well as their amplitudes are constant (constant force along the actuators stroke), the resulting grasping force does not vary.

The performances of the manipulator could be enhanced by optimizing the design parameters. Besides, the design of the fingers could also be optimized to obtain the desired elasticity at the fingertips to ensure an homogeneous grasping force of the object. 




Figure 10. CAD model of the spherical joint made with a ruby.

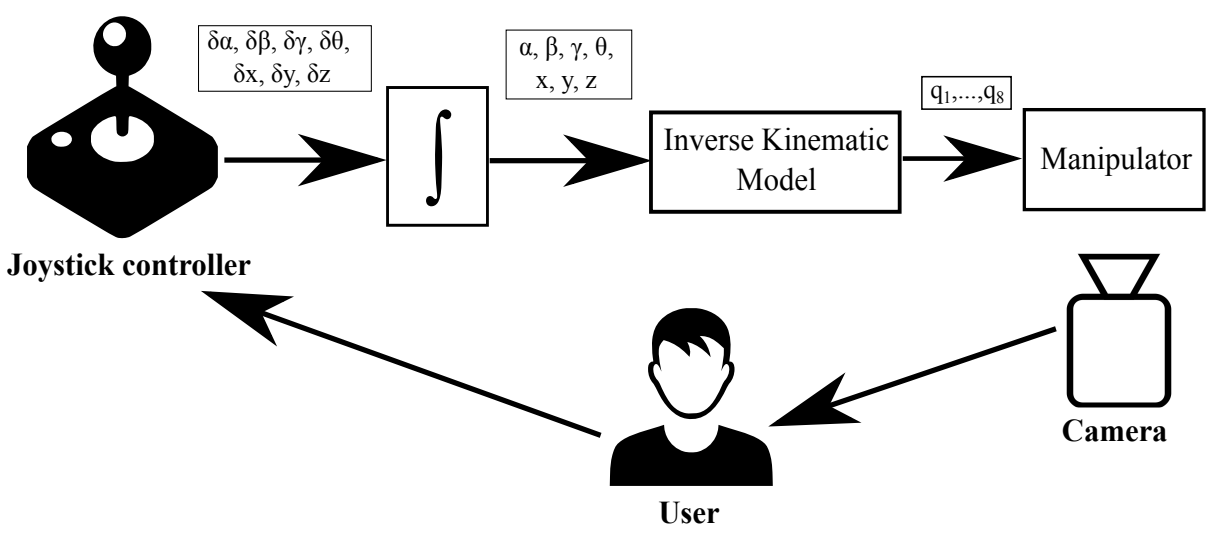

Figure 11. Schematic representation of the experimental setup.

\section{Experimental Validation}

An experimental prototype was constructed and tested. The prototype was designed for manipulation and assembly applications. The eight struts were made of aluminum rods of $3 \mathrm{~mm}$ in diameter and $50 \mathrm{~mm}$ in length. Ball-and-socket spherical joints were used in the prototype. In order to reduce frictions and backlash, the ball-insocket technology was used where the balls were made of ruby. Each ball is inserted in a notch and held between two coaxial through bores. Fig. 10 shows a drawing of the used spherical joint.

In this first prototype, each of the eight mobile bases was actuated by an $18 \mathrm{~mm}$ travel motion and $1 \mu \mathrm{m}$ repeatability Smaract SLC-1730 piezoelectric actuators fixed vertically to the base.

The purpose of these experiments is to validate the proposed robotics structure through grasping, manipulating, and insertion operations of a millimeter scale object. To do so, the manipulator was controlled manually using a joystick. The inverse kinematic model of the robot was used to calculate the eight joint values from the desired Cartesian trajectories that are required by the user through the joystick as illustrated in Fig. 11.

In these experiments, an object of $2 \mathrm{~mm}$ in diameter and $10 \mathrm{~mm}$ in length is manipulated. First, the gripper is correctly positioned above the object and the robot's platform is folded to grasp it. Second, the object is moved-up from its hole and manipulated in the six DoFs. Finally, the object is inserted in the $2.4 \mathrm{~mm}$ in diameter target hole and released (see Fig. 12). The manipulation process can be further appreciated in the video clip accompanying this paper.

This experiment demonstrates the ability of the robot to grasp and to manipulate an object with Cartesian reference trajectories generated from the joystick and transformed to joint space trajectories. Initially, the design parameters listed in Table 1 were implemented in the kinematic model of the prototype. However, high internal stresses in the structure appeared due to fabrication and assembly errors.

Thus, a pre-calibration phase was done thanks to the actuaction and measurement redundancies. Thanks to this pre-calibration phase, the internal stress in the robot's structure was reduced and the executed trajectories were greatly improved. Nevertheless, an experimental evaluation of the repeatability and the accuracy of the manipulator using an external measurement system is left to future works. 


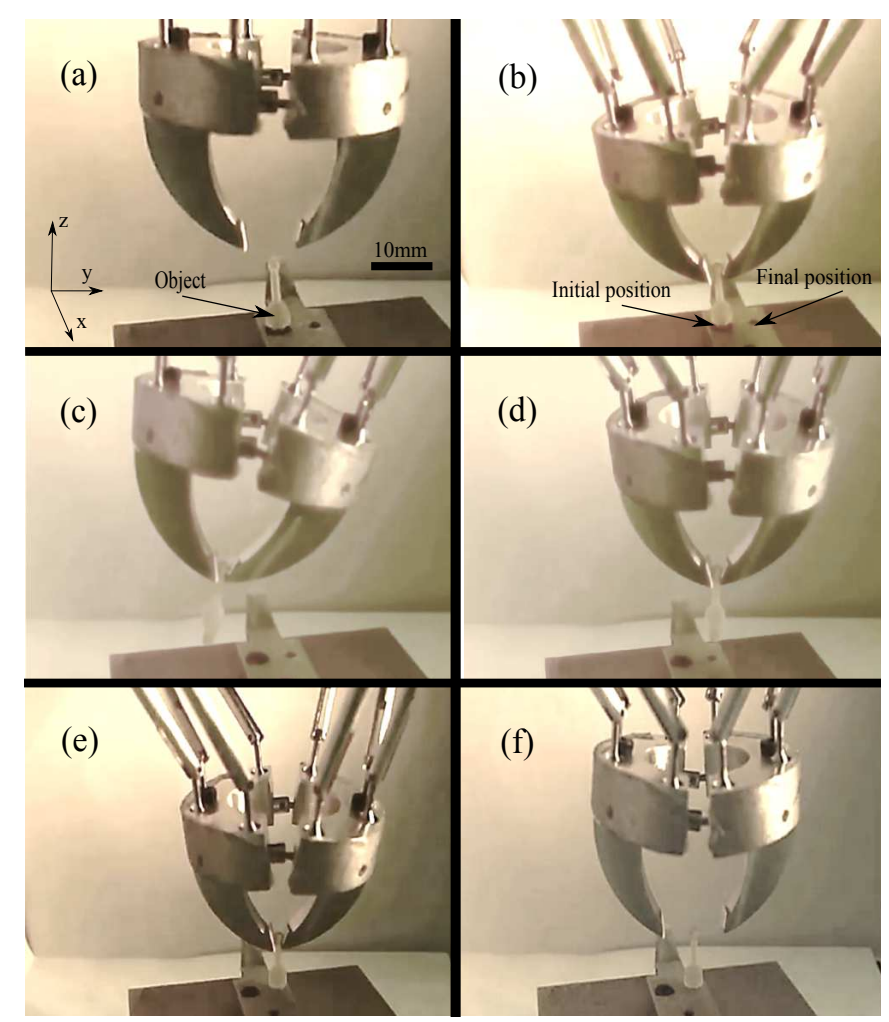

Figure 12. Manipulation and insertion of an object. (a) Object initially inserted in the first hole. (b) Positioning the end-effector and grasping of the object. (c) Manipulating the object with the different DoFs. (d) Positioning the object to the target position. (e) Inserting the object. (f) Releasing the object.

\section{Discussion}

The presented manipulator is a $7 \mathrm{DoF}$ redundant parallel robot with a configurable platform. The robot platform can be positioned in the $6 \mathrm{DoF}$ and used for grasping, cutting, or any other operation which can be obtained by a folding mechanism.

One of the main advantages of this structure is its compactness thanks to all the actuators located on the robot base. In addition, all the eight actuators contribute to the force applied by the platform thanks to the redundant parallel structure. Relatively, high forces and torques can then be applied by the platform as shown in Fig. 9.

The forces are transmitted to the platform through eight links which work in traction/compression. This configuration makes the structure stiff compared to its mass, inertia, and volume. High dynamics could then be reached with appropriate actuators, such as linear voice coils which can achieve more than $200 \mathrm{~m} / \mathrm{s}^{2}$ accelerations [29].

The variability of the grasping force within its workspace should be taken into account when integrating the robot. Since one can take advantage of the eventually high forces applied by the robot's actuators, controlling the grasping force could be a strategy to leverage the effect of this drawback. The grasping force control could thus be done in several ways.

For instance, in manipulation applications, one part of the platform could be equipped with an articulated end-effector and pre-loaded thanks to a spring. Such a mechanism would allow controlling the position of the manipulated object (thanks to the rigid end-effector) as well as the grasping force thanks to the articulated endeffector. Indeed, in this case, the grasping force can be controlled through the robot jaw closing (more the platform closes more the force is applied on the end-effector thanks to the spring).

In applications where fine force control is required (cells handling, for instance), the integration of force sensing associated with appropriate actuators whose applied forces can be controlled could also be a solution. Finally, in shearing, cutting and demolition applications, rigid end-effectors could be used since no force control in these applications is usually required.

The structure is symmetric thanks to the eight actuators which improves the homogeneity of the robot and simplifies its modeling and design. The actuation redundancy is not directly exploited in this paper. Nevertheless, it will be exploited in different ways in future works.

First of all, the robot joints positions will be optimized to reduce the singular configurations and enlarge the 
robot workspace. This can be done thanks to the kinematic singularities analysis of the robot using screw theory and Grassmann-Cayley algebra (GCA) [30]. To do so, existing methods will have to be adapted to this class of robots because of the 7 DoF configurable platform (more than 6 DoF), whose motion cannot be represented by a Spatial Euclidian Group element (SE3).

One of the drawbacks of redundant actuation is the possible existence of internal stress in the robot structure. This can be avoided by implementing hybrid force position control laws [11] thanks to position and force sensors, placed on the robot actuators for instance. The redundant measurements could also be used to simplify the identification of the kinematic model of the robot thanks to auto-calibration techniques [31] [32].

Several applications can be considered. One of the major domains where dexterity, compactness and high blocking forces are required is robotic surgery [33] [34]. Indeed, the configuration platform can be used for grasping and cutting thanks to appropriate tools fixed on the foldable platform. In this case, the actuators can be placed outside the body and actuate the structure thanks to rods, or cables associated for instance with pre-constrained springs to keep the cables stretched [35]. Thus, all the $7 \mathrm{DoF}$ can be exploited inside the body contrarily to existing technologies where the DoF are generated outside the body and constrained by the insertion hole [36].

Another possible application is micro/nano-manipulation. At the micro/nano-scale, it is almost impossible to attach a gripper on a micro-robotic structure because of miniaturization limits [16]. The solution usually adopted is to use a parallel micro-robot as a 6 DoF positioning table associated with an external static or moving gripper [37] [38]. This configuration is more complex and not feasible in some applications such as in cells manipulation.

The proposed 7 DoF structure can be highly miniaturized using flexure hinges instead of spherical joints [7] [9]. The micro-robot can then have an integrated gripper and work as a complete 7 DoF micro-robot for dexterous manipulation and benefit from high precision linear actuation technologies.

Other applications can be considered where high forces have to be applied. Typical applications would be hydraulic clamps or shears with 3D position and orientation control which can be used for construction/destruction robotics and heavy industries (shipbuilding, aeronautics, nuclear, etc.) [1].

\section{Conclusion}

In this paper, we proposed a new robotic structure with a configurable platform. This robot has six-DoFs manipulation and grasping capabilities using linear stages located on the robot's base. The robot structure was investigated and its kinematic model developed. For the proof of concept, a prototype was fabricated and controlled. This robot was able to successfully achieve grasping, manipulation, and insertion operations which validates the proposed concept. Further developments of the robot will focus on the singularity analysis of the proposed structure, design optimization and, control exploiting actuation and sensing redundancy, which will enhance the robot capabilities and performances.

\section{Acknowledgments}

This work was supported by the Région de Bourgogne-Franche-Comté, Labex ACTION project (ANR-11LABX-01-01), Equipex ROBOTEX project (ANR-10-EQPX-44-01) and the French "Investissements d'Avenir" program, project ISITE-BFC Robotics Nanofactory (ANR-15-IDEX-03).

The authors gratefully acknowledge Vincent Tissot and the mechanics service from the FEMTO-ST Institute for their technical assistance.

\section{References}

[1] Merlet, J.-P., 2012. Parallel robots, Vol. 74. Springer Science \& Business Media.

[2] GOUGH, V. E., 1957. "Contribution to discussion to papers on research in automobile stability and control and in tire performance". Proc. of the Automotive Division of the Institute of Mechanical Engineers, 1957, 180, pp. 392-394.

[3] Pierrot, F., Reynaud, C., and Fournier, A., 1990. "Delta: a simple and efficient parallel robot". Robotica, 8(02), pp. 105-109.

[4] Li, Y., Ma, Y., Liu, S., Luo, Z., Mei, J., Huang, T., and Chetwynd, D., 2014. "Integrated design of a 4-dof high-speed pick-and-place parallel robot". CIRP Annals-Manufacturing Technology, 63(1), pp. 185-188.

[5] Xie, F., and Liu, X.-J., 2015. "Design and development of a high-speed and high-rotation robot with four identical arms and a single platform". Journal of Mechanisms and Robotics, 7(4), p. 041015. 
[6] Ozgur, E., Dahmouche, R., Andreff, N., and Martinet, P., 2013. "High speed parallel kinematic manipulator state estimation from legs observation". In Intelligent Robots and Systems (IROS), 2013 IEEE/RSJ International Conference on, IEEE, pp. 424-429.

[7] Brouwer, D., De Jong, B., and Soemers, H., 2010. "Design and modeling of a six dofs mems-based precision manipulator". Precision Engineering, 34(2), pp. 307-319.

[8] Shi, H., Su, H.-J., and Dagalakis, N., 2014. "A stiffness model for control and analysis of a MEMS hexapod nanopositioner". Mechanism and Machine Theory, 80, pp. 246-264.

[9] Yao, Q., Dong, J., and Ferreira, P. M., 2008. "A novel parallel-kinematics mechanisms for integrated, multiaxis nanopositioning: Part 1. kinematics and design for fabrication". Precision Engineering, 32(1), pp. 7-19.

[10] Shi, H., and Su, H.-J., 2013. "An analytical model for calculating the workspace of a flexure hexapod nanopositioner". Journal of Mechanisms and Robotics, 5(4), p. 041009.

[11] Müller, A., 2008. Redundant Actuation of Parallel Manipulators, Parallel Manipulators, towards New Applications. INTECH Open Access Publisher.

[12] Kim, J., Park, F. C., Ryu, S. J., Kim, J., Hwang, J. C., Park, C., and Iurascu, C. C., 2001. "Design and analysis of a redundantly actuated parallel mechanism for rapid machining". IEEE Transactions on Robotics and Automation, 17(4), pp. 423-434.

[13] Alba, O., Pamanes, J., and Wenger, P., 2007. "Trajectory planning of a redundant parallel manipulator changing of working mode". In 12th IFToMM World Congress, Besancon (France), pp. 18-21.

[14] O'Brien, J. F., and Wen, J. T., 1999. "Redundant actuation for improving kinematic manipulability". In Robotics and Automation, 1999. Proceedings. 1999 IEEE International Conference on, Vol. 2, IEEE, pp. 15201525.

[15] Luces, M., Mills, J. K., and Benhabib, B., 2017. "A review of redundant parallel kinematic mechanisms". Journal of Intelligent \& Robotic Systems, 86(2), pp. 175-198.

[16] Gauthier, M., and Régnier, S., 2011. Robotic micro-assembly. John Wiley \& Sons.

[17] Pierrot, F., and Company, O., 1999. "H4: A new family of 4-dof parallel robots". In Advanced Intelligent Mechatronics, 1999. Proceedings. 1999 IEEE/ASME International Conference on, IEEE, pp. 508-513.

[18] Zhang, D., 2009. Parallel robotic machine tools. Springer Science \& Business Media.

[19] Hoevenaars, A. G., Lambert, P., and Herder, J. L., 2014. "Kinematic design of two elementary 3dof parallel manipulators with configurable platforms". In Computational Kinematics. Springer, pp. 315-322.

[20] Nabat, V., de la O, R., María, O., Company, O., Krut, S., and Pierrot, F., 2005. "Par4: very high speed parallel robot for pick-and-place". In IEEE/RSJ International Conference on Intelligent Robots and Systems, pp. 553-558.

[21] Yi, B.-J., Na, H. Y., Lee, J. H., Hong, Y.-S., Oh, S.-R., Suh, I. H., and Kim, W. K., 2002. "Design of a parallel-type gripper mechanism". The International Journal of Robotics Research, 21(7), pp. 661-676.

[22] Mohamed, M. G., and Gosselin, C. M., 2005. "Design and analysis of kinematically redundant parallel manipulators with configurable platforms". in IEEE Transactions on Robotics, 21(3), pp. 277-287.

[23] Lambert, P., and Herder, J. L., 2014. Computational Kinematics: Proceedings of the 6th International Workshop on Computational Kinematics (CK2013). Springer Netherlands, Dordrecht, ch. Self Dual Topology of Parallel Mechanisms with Configurable Platforms, pp. 291-298.

[24] Lambert, P., and Herder, J., 2015. "A novel parallel haptic device with 7 degrees of freedom". In IEEE World Haptics Conference (WHC), Chicago, pp. 183-188.

[25] Haouas, W., Dahmouche, R., Le Fort-Piat, N., and J. Laurent, G., 2016. "4-DoF spherical parallel wrist with embedded grasping capability for minimally invasive surgery". In International Conference on Intelligent Robots and Systems.

[26] Stoughton, R. S., and Arai, T., 1993. "A modified stewart platform manipulator with improved dexterity". IEEE Transactions on Robotics and Automation, 9(2), pp. 166-173.

[27] Pusey, J., Fattah, A., Agrawal, S., and Messina, E., 2004. "Design and workspace analysis of a 6-6 cablesuspended parallel robot". Mechanism and machine theory, 39(7), pp. 761-778.

[28] Wen, J.-Y., and Wilfinger, L. S., 1999. "Kinematic manipulability of general constrained rigid multibody systems". IEEE Transactions on Robotics and Automation, 15(3), pp. 558-567.

[29] Wu, S., Jiao, Z., Yan, L., Zhang, R., Yu, J., and Chen, C.-Y., 2014. "Development of a direct-drive servo valve with high-frequency voice coil motor and advanced digital controller". IEEE/ASME Transactions on Mechatronics, 19(3), pp. 932-942.

[30] Amine, S., Masouleh, M. T., Caro, S., Wenger, P., and Gosselin, C., 2012. "Singularity conditions of 3t1r parallel manipulators with identical limb structures". Journal of Mechanisms and Robotics, 4(1), p. 011011.

[31] il Jeong, J., Kang, D., Cho, Y. M., and Kim, J., 2004. "Kinematic calibration for redundantly actuated parallel mechanisms". Journal of Mechanical Design, 126(2), pp. 307-318.

[32] Valasek, M., Belda, K., and Florian, M., 2002. "Control and calibration of redundantly actuated parallel 
robots". In 3rd Parallel Kinematics Seminar Chemnitz, Chemnitz, pp. 411-427.

[33] Abbott, D. J., Becke, C., Rothstein, R. I., and Peine, W. J., 2007. "Design of an endoluminal notes robotic system". In Intelligent Robots and Systems, 2007. IROS 2007. IEEE/RSJ International Conference on, IEEE, pp. $410-416$.

[34] Pryor, A. D., Tushar, J. R., and DiBernardo, L. R., 2010. "Single-port cholecystectomy with the transenterix spider: simple and safe". Surgical endoscopy, 24(4), pp. 917-923.

[35] Kaouk, J. H., Goel, R. K., Haber, G.-P., Crouzet, S., and Stein, R. J., 2009. "Robotic single-port transumbilical surgery in humans: initial report". BJU international, 103(3), pp. 366-369.

[36] Niccolini, M., Petroni, G., Menciassi, A., and Dario, P., 2012. "Real-time control architecture of a novel singleport laparoscopy bimanual robot (sprint)". In Robotics and Automation (ICRA), 2012 IEEE International Conference on, IEEE, pp. 3395-3400.

[37] Petrovic, D., Chatzitheodoridis, E., Popovic, G., Del Medico, O., Almansa, A., Brenner, W., Detter, H., Martins, R., and Fortunato, E., 2001. Design of a mechanical gripper for assembly of microparts. na.

[38] Popa, D. O., and Stephanou, H. E., 2004. "Micro and mesoscale robotic assembly". Journal of manufacturing processes, 6(1), pp. 52-71. 


\section{List of Figures}

1 Architectural scheme of the manipulator. The blocks P, R, and S represent prismatic, revolute, and spherical joints, respectively. . . . . . . . . . . . . . . . . . . . . . 3

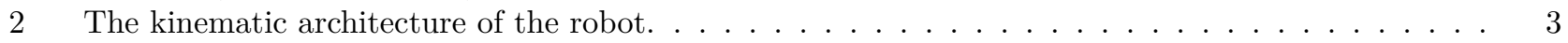

3 Geometrical description of the top platform and bottom stages. . . . . . . . . . . . . . . 6

4 Kinematic design parameters h1, h2, h3 and h4. . . . . . . . . . . . . . . . 6

$5 \quad$ Manipulability index of the manipulator at its home position as a function of $\phi_{1}$ and $\phi_{2} \ldots \ldots$

6 Representation of the volume of the workspace $\left(\mathrm{cm}^{3}\right)$ of the manipulator as a function of $\phi_{1}$ and $\phi_{2} \quad 7$

7 Theoretical workspace of the two fingers of the manipulator using $18 \mathrm{~mm}$ travel range actuators. . 8

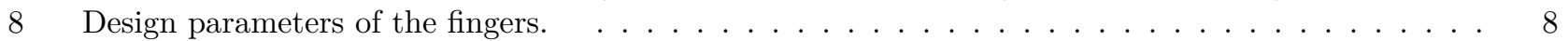

9 Maximal grasping couple over poses range (N.m). . . . . . . . . . . . . . . . . . . . 9

10 CAD model of the spherical joint made with a ruby. . . . . . . . . . . . . . . . . . . . . 10

11 Schematic representation of the experimental setup. . . . . . . . . . . . . . . . 10

12 Manipulation and insertion of an object. (a) Object initially inserted in the first hole. (b) Positioning the end-effector and grasping of the object. (c) Manipulating the object with the different DoFs. (d) Positioning the object to the target position. (e) Inserting the object. (f) Releasing the object.

\section{List of Tables}

1 Kinematic Design Parameters. . . . . . . . . . . . . . . . . . . . . .

2 Maximum displacement and rotation angles of the center of the foldable platform using $18 \mathrm{~mm}$ travel motion of the actuators. . . . . . . . . . . . . . . . . . . . . . . . 